\title{
Laparoscopic Radical Cystectomy With Intracorporeal Orthotopic Neobladder Reconstruction: An Initial Experience of Modified Studer Reconstruction Techniques
}

\author{
Jeong Ho Kim \\ Department of Urology, Inje University Haeundae Paik Hospital, Inje University College of Medicine, Busan, Korea
}

\begin{abstract}
This study aimed to describe the initial experience of laparoscopic radical cystectomy (LRC) with total intracorporeal urinary diversion (ICUD). The procedure was performed in a male patient using a 5-port transperitoneal approach. Radical cystoprostatectomy and bilateral pelvic lymphadenectomy were performed laparoscopically. A 40-cm segment of the ileum $20 \mathrm{~cm}$ from the ileocecal junction was then isolated, and ileo-ileal continuity was restored using EndoGIA staplers. Bilateral ureteroileal anastomoses were individually performed using the modified Studer chimney. Running urethroileal anastomosis was performed the midpoint of the remaining distal $30 \mathrm{~cm}$ of the ileal segment. The distal $30 \mathrm{~cm}$ of the isolated ileal segment was detubularized, and a modified Studer neobladder was constructed. The total operative time was 465 minutes, and the estimated blood loss was $890 \mathrm{~mL}$. The patient resumed ambulation on postoperative day 2 , and oral liquids were resumed on postoperative day 8 . The surgical margins of bladder specimens were negative for cancer. LRC with ICUD performed completely intracorporeally was feasible. Large-scale studies are needed to define these benefits and corroborate these findings.
\end{abstract}

Key Words: Cystectomy, Intracorporeal, Bladder cancer
Received February 16, 2021

Revised March 15, 2021

Accepted March 30, 2021

Corresponding author:

Jeong Ho Kim

Email: saleen81@naver.com

https://orcid.org/0000-0003-4491-0308
Advances in cancer surgery have enabled more delicate resection of tumors and have minimized harm to patients. Technological progress in minimally invasive surgery has resulted in significant benefits for patients, such as reduced blood loss and postoperative pain, decreased postoperative morbidity, shorter duration of hospital stay, and early return to normal activities. Robotic surgery is rapidly replacing conventional laparoscopic surgery in the abdominopelvic field. In particular, the clinical application of robotic surgery has expanded from malignancies to benign diseases in urology. Although the definite advantages of robotic surgery have been controversial, several studies have reported that robotic surgery has more benefits than conventional laparoscopic or open surgery. However, the cost is a serious issue because robotic surgery is not covered by the national health service. It is also generally more expensive than other minimally invasive surgeries, especially in Korea. Although conventional laparoscopic surgery has a steeper learning curve than robotic surgery, it still has the potential to treat patients who require this type of surgery and utilize the national health service.

Radical cystectomy is the standard treatment for muscle-invasive bladder cancer, and diverse surgical procedures have been performed for urinary 
diversion. In recent years, radical cystectomy has been frequently performed using a robot-assisted system, and robot-assisted total intracorporeal urinary diversion (ICUD) has been performed in high-volume centers. The benefits of total ICUD surgery are to minimize bowel manipulation and exposure to air. However, conventional laparoscopic ICUD was introduced about 20 years ago, but it did not become part of general practice because of the difficulty and technical complexity of the procedure. ${ }^{1}$ In addition, there were concerns regarding the safety of the procedure; therefore, most laparoscopic neobladder reconstructions were performed extracorporeally. However, there are also some advantages to laparoscopic ICUD. This study aimed to describe an initial experience of laparoscopic radical cystectomy (LRC) with total ICUD.

\section{CASE REPORT}

A 64-year-old man consulted our hospital due to of painless hematuria. The patient had been on insulin for 20 years, and his blood glucose level was not well-controlled. After admission, the initial glycosylated hemoglobin level was $7.3 \%$. He is also a heavy smoker for over 44 years. A computed tomography (CT) scan showed a bladder tumor measuring $7 \mathrm{~cm}$ in the left posterolateral aspect of the bladder wall with multiple small tumors, and accompanied by a mild left hydroureter. A large papillary tumor with a broad base and multiple small tumors were observed on cystoscopy. Metastatic lesions or lymph node enlargement were not detected by chest and abdominopelvic CT scan; therefore, the patient was diagnosed with a clinical T3N0M0 bladder tumor. The patient underwent transurethral resection of the bladder tumor. Histopathological analysis showed an infiltrating urothelial carcinoma with invasion into the muscle layer. Neoadjuvant chemotherapy with
ddMVAC and radical cystectomy with neobladder reconstruction were planned. He tolerated 4 cycles of chemotherapy well. Radical cystectomy with a modified Studer ileal neobladder was subsequently performed using a total laparoscopic intracorporeal technique. The total operative time and intracorporeal neobladder reconstruction time were 7 hours 45 minutes and 4 hours 2 minutes, respectively. The estimated blood loss was $890 \mathrm{~mL}$. There were no intraoperative complications. The final histopathological results showed infiltrating urothelial carcinoma with squamous differentiation and perivesical tissue invasion. All the surgical margins were negative. No cancer cells were detected in the resected lymph nodes. Although postoperative paralytic ileus persisted for 7 days, the patient fully recovered without any additional surgery.

\section{Procedure}

All surgical procedures were performed as described in previous studies. ${ }^{2}$ The patient was placed in lithotomy position with a steep Trendelenburg slope. Pneumoperitoneum was created using a Veress needle via a supraumbilical site, and the abdomen was insufflated to $15 \mathrm{mmHg}$. The camera port was placed just above the umbilicus, and the other 4 ports were inserted under direct vision. After bilateral pelvic lymphadenectomy, cystoprostatectomy was performed. Cystoprostatectomy was initiated by incising the peritoneum transversely at the recto-vesical cul-de-sac to identify the posterior plane between the bladder and the rectum. After both seminal vesicles were identified, the dissection proceeded down to Denonvilliers' fascia. The Denonvilliers' fascia was incised horizontally to reveal the perirectal fat. This plane was followed distally towards the apex of the prostate. Both ureters were ligated and divided close to the bladder. The peritoneal inci- 
sion was made along the bilateral medial umbilical ligaments, and the dissection was continued to enter the Retzius space and identify the endopelvic fascia. The endopelvic fascia was opened bilaterally, and the dorsal vein complex was ligated. The lateral and posterior vascular pedicles of the bladder and prostate were controlled using vascular clips and laparoscopic bipolar electrosurgical device. The prostate and bladder were then carefully separated from the urethra to preserve maximum urethral length and the urethral sphincter. A window was made under the sigmoid mesocolon, and the left ureter was transposed to the right side through the window. The specimens were placed in a laparoscopic retrieval bag. Finally, cystoprostatectomy was performed. The next step was the creation of a neobladder, for which the terminal ileum was identified.

Neobladder creation was planned by making a modified U-Shaped ileal neobladder (Fig. 1). As a first step, 3 marking sutures were placed to harvest the distal small bowel. The first suture was placed $20 \mathrm{~cm}$ proximal to the cecum. The second suture was placed $20 \mathrm{~cm}$ proximal from the first suture, and the third suture was made $20 \mathrm{~cm}$ proximal from the second suture. A $20-\mathrm{cm}$ suture was in-

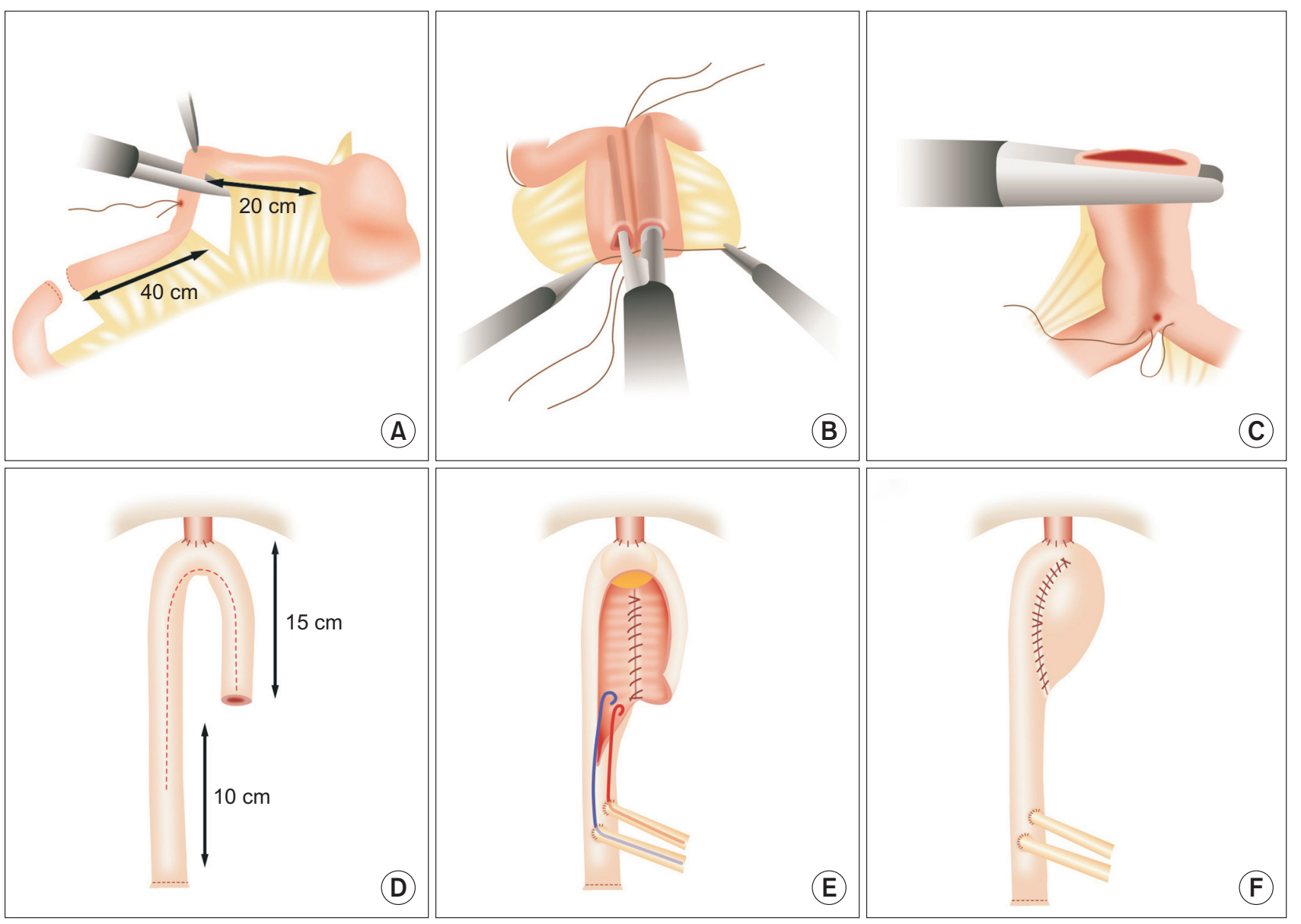

Fig. 1. Illustration of surgical procedures demonstrates reconstruction of neobladder. (A) The distal $40 \mathrm{~cm}$ of the terminal ileum was isolated using laparoscopic Endo-GIA with a 60-mm intestinal stapler. (B) Bowel continuity was restored with side-to-side anastomosis by stapler. (C) Open ends of the ileal stump closed using another stapler. (D) The urethroileal anastomosis was performed with a running suture with barbed sutures. A chimney of the neobladder was made measuring $10 \mathrm{~cm}$ in length. (E) Each ureter was separately anastomosed to the chimney of the neobladder. A double-J stent was placed each ureter. The posterior wall was constructed after detubularization of the isolated ileum. (F) The anterior wall was closed to complete construction of the neobladder. 
troduced into the abdomen and was used to measure the length of bowel. The ileum was divided with laparoscopic Endo-GIA staplers (COVIDIEN, Tokyo, Japan; staplers $60 \mathrm{~mm}$, purple cartridge) guided by the previously placed sutures (Fig. 2A). The total length of the isolated small bowel was $40 \mathrm{~cm}$. The harvested ileal segment was placed posteriorly, and side-to-side ileal anastomosis was performed with laparoscopic Endo-GIA staplers, restoring the ileal continuity (Fig. 2B). The proximal $10 \mathrm{~cm}$ of the isolated ileal segment was maintained for the isoperistaltic Studer chimney. The harvested ileal segment was delivered into the pelvic cavity toward the urethral stump, with the midpoint of the remaining distal $30 \mathrm{~cm}$ of the ileal segment selected for performing the anastomosis. A running urethroileal anastomosis was performed using two 3-0 barbed sutures (Fig. 2C). A 20F 3way Foley catheter was inserted through the urethra and into the isolated ileal segment after completing the urethroileal anastomosis. Two small ileotomy were created on the Studer chimney for ureteroenteric anastomosis. Both ureteral ends were freshened and spatulated for anastomosis, and a 6F 28-cm double-J catheter was inserted into the lumen (Fig. 2D). Ureteroenteric anastomosis was performed with full-thickness, singlelayer sutures using 4-0 multifilament absorbable sutures. The remaining distal $30 \mathrm{~cm}$ of the ileal segment was detubularized along the antimesenteric border using surgical energy devices (Fig. 2E). The posterior and anterior walls of the neobladder were created by continuous suturing of the corresponding edges of the detubularized ileal segment using 3-0 barbed sutures (Fig. 2F). The neobladder was irrigated through a Foley catheter, and leak sites were carefully sutured. A Jackson-Pratt drain was placed in the pelvis through the lateral port site. The entire surgical procedure was performed intracorporeally.

\section{Perioperative Outcomes}

The total operation time was 465 minutes, and the estimated blood loss was $890 \mathrm{~mL}$. The patient did not require blood transfusion and resumed ambulation on postoperative day 2. Oral liquids were resumed only on postoperative day 8 be-
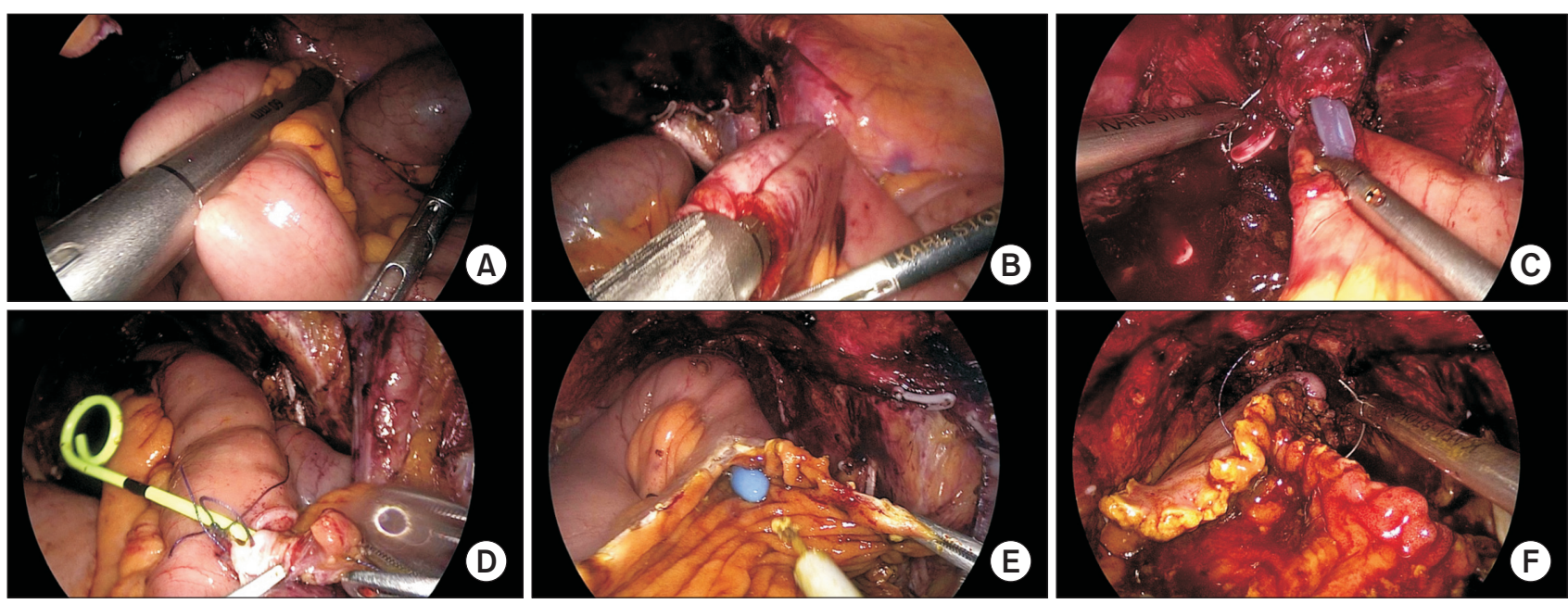

Fig. 2. Images of laparoscopic completely intracorporeal modified Studer neobladder techniques. (A) The distal end of the selected ileal segment was transected using an Endo-GIA stapler. (B) Side-to-side anastomosis of the 2 ends of the ileum was performed using an Endo-GIA stapler. (C) The urethroileal anastomosis was performed using running barbed sutures. (D) The ureteroileal anastomosis was performed, and double-J ureteral stents were inserted. (E) The isolated ileum was detubularized using surgical energy devices. (F) The anterior wall of the orthotopic neobladder was sutured, and the spherical neobladder was completely constructed. 
cause postoperative ileus persisted for 7 days. It was resolved with supportive care including ambulation, hydration, glycerin, and finger enema. The duration of hospital stay was 21 days. Histopathological analysis revealed pT3 high-grade urothelial carcinoma. The resected lymph nodes and surgical margins of the bladder specimens were all negative for malignancy.

\section{Ethics Approval Statement}

This study was approved by the Institutional Review Board of the Inje University Haeundae Paik Hospital (HPIRB 2020-11-008) and was conducted according to the ethical standards laid down by the 1964 Declaration of Helsinki and its later amendments.

\section{DISCUSSION}

Radical cystectomy is the gold standard treatment for muscle-invasive bladder cancer. When the bladder is resected, urinary diversion is required to store and eliminate urine from the body. There are several different types of urinary diversion, with the ileal conduit being the most widely employed. The ileal conduit urinary diversion has some disadvantages, such as decreased quality of life due to an external urostomy, and the use of an external bag to collect urine, which may leak and have foul-smelling odors. Therefore, most patients are reluctant to undergo the ileal conduit operation. Moreover, a previous study reported that patients with orthotopic neobladder substitution have a better quality of life and a more active lifestyle compared to patients with an ileal conduit. ${ }^{3}$ However, urologic surgeons generally do not prefer orthotopic neobladder substitution surgeries because they require a longer operating time, are technically more difficult, and tend to have higher complication rates. In addition, for orthotopic neobladder substitution, it is necessary to consider strict indications and contraindications. ${ }^{4}$ Therefore, each urinary diversion method has specific advantages and disadvantages with respect to perioperative and functional outcomes that must be considered while selecting the best option for each individual patient.

After the initial report of LRC, surgical techniques were developed remarkably with the modification of surgical instruments. ${ }^{5}$ Several studies comparing open radical cystectomy (ORC) and LRC have reported no significant differences in oncologic outcomes. ${ }^{6}$ LRC has some advantages, such as improved cosmesis, pain control, and less blood loss, but these are not enough for a paradigm shift. Therefore, the choice of surgical access technique was decided based on patient counseling and the surgeon's preference. In the last decade, robotic surgery has been widely used, but despite the lack of significant benefits over ORC, the proportion of robot-assisted radical cystectomy (RARC) has increased steadily over the years. After RARC became more widely accepted, total ICUD started to regain attention. Simone et al. ${ }^{7}$ reported a significantly lower incidence of perioperative transfusion rates in the group of patients who had RARC with ICUD. The reduced intraoperative blood loss, which consequently minimized the requirement of perioperative transfusions, was also found to have a positive effect on survival outcomes. To date, there has been no large-scale study on LRC with ICUD, though recent smaller studies have demonstrated that LRC with ICUD was effective in reducing postoperative morbidity. ${ }^{8}$ The studies reporting clinical outcomes of LRC with ICUD are summarized in Table 1.,5,8-10 Although this study is a report of an initial experience, perioperative outcomes could be expected to improve further as experience is increased. Laparoscopic surgery has evolved over time with the introduction of new surgical techniques and 
Table 1. Summary of clinical outcomes of review studies

\begin{tabular}{|c|c|c|c|c|c|c|c|c|}
\hline Study & $\begin{array}{c}\text { Year of } \\
\text { publication }\end{array}$ & $\begin{array}{c}\text { No. of } \\
\text { patients }\end{array}$ & Age (yr) & $\begin{array}{c}\text { Neobladder } \\
\text { type }\end{array}$ & $\begin{array}{l}\text { Total } \\
\text { operative time } \\
\text { (min) }\end{array}$ & $\begin{array}{l}\text { Time of neobladder } \\
\text { reconstruction } \\
\text { (min) }\end{array}$ & $\mathrm{EBL}(\mathrm{mL})$ & $\begin{array}{l}\text { Length of } \\
\text { hospital stay } \\
\text { (day) }\end{array}$ \\
\hline Gill et al. ${ }^{1}$ & $\begin{array}{c}2002 \\
\text { (initial report) }\end{array}$ & 2 & N/A & Studer & $\mathrm{N} / \mathrm{A}$ & N/A & N/A & N/A \\
\hline Shao et al. ${ }^{9}$ & 2014 & 55 & $58.5 \pm 9.0$ & U-shaped & $346 \pm 52$ & $230 \pm 41.9$ & $500(250-1,400)$ & N/A \\
\hline $\begin{array}{l}\text { Pastore } \\
\text { et al. }^{8}\end{array}$ & 2014 & 30 & $67(62-79)$ & U-shaped & $365(270-605)$ & N/A & $290(70-800)$ & 9 \\
\hline $\begin{array}{l}\text { Bujoreanu } \\
\text { et al. }\end{array}$ & 2020 & 23 & N/A & Y-shaped & 300 & $150 \pm 7$ & $350 \pm 40$ & $21 \pm 3$ \\
\hline $\mathrm{Xu}$ et al. ${ }^{10}$ & 2020 & 12 & $54.8 \pm 17.6$ & $\begin{array}{r}\text { Detaenial } \\
\text { sigmoid }\end{array}$ & $414.6 \pm 52.2$ & $33.8 \pm 6.8$ & $217.5 \pm 40.0$ & $11.8 \pm 2.27$ \\
\hline
\end{tabular}

Values are presented as mean \pm standard deviation or median (range).

EBL: estimated blood loss, N/A: not available.

instruments, such as single-port surgery, barbed suture, and 3-dimensional laparoscopy. Laparoscopic surgery has the advantage of lower cost compared to robotic surgery and has the benefits of cosmesis and pain control compared to open surgery. Therefore, it warrants further research and attention.

In conclusion, LRC with ICUD can be effectively performed by total intracorporeal techniques in a manner duplicating open surgical procedures. This initial experience suggests that LRC with ICUD is a feasible and cost-effective option for muscle-invasive bladder cancer. Long-term largescale studies are required to assess the clinical outcomes.

\section{CONFLICT OF INTEREST}

The author claims no conflicts of interest.

\section{REFERENCES}

1. Gill IS, Kaouk JH, Meraney AM, Desai MM, Ulchaker JC, Klein EA, et al. Laparoscopic radical cystectomy and continent orthotopic ileal neobladder performed completely intracorporeally: the initial experience. J Urol 2002;168:13-8.

2. Kang SG, Ko YH, Jang HA, Kim J, Kim SH, Cheon
$\mathrm{J}$, et al. Initial experience of robot-assisted radical cystectomy with total intracorporeal urinary diversion: comparison with extracorporeal method. J Laparoendoscopic Adv Surg Tech A 2012;22:45662.

3. Philip J, Manikandan R, Venugopal S, Desouza J, Javlé PM. Orthotopic neobladder versus ileal conduit urinary diversion after cystectomy--a qualityof-life based comparison. Ann R Coll Surg Engl 2009;91:565-9.

4. Minervini A, Serni S, Vittori G, Masieri L, Siena G, Lanciotti $\mathrm{M}$, et al. Current indications and results of orthotopic ileal neobladder for bladder cancer. Expert Rev Anticancer Ther 2014;14:419-30.

5. Bujoreanu CE, Rusu CB, Irimie A, Achimas-Cadariu P, Vlad C, Kubelac P, et al. Totally intracorporeal 3D laparoscopic orthotopic neobladder reconstruction following radical cystectomy for infiltrative bladder tumors: the experience of modified Studer and modified Y-shaped ileal reconstruction techniques. J BUON 2020;25:286-94.

6. Çelen S, Kaygisız O, Vuruşkan H, Yavaşcaoğlu İ. Laparoscopic versus open radical cystectomy in the treatment of locally advanced T3 and T4 bladder cancer: perioperative and mid-term oncological outcomes. Turk J Urol 2020;46:123-8.

7. Simone G, Tuderti G, Misuraca L, Anceschi U, Ferriero $\mathrm{M}$, Minisola $\mathrm{F}$, et al. Perioperative and mid- 
term oncologic outcomes of robotic assisted radical cystectomy with totally intracorporeal neobladder: results of a propensity score matched comparison with open cohort from a single-centre series. Eur J Surg Oncol 2018;44:1432-8.

8. Pastore AL, Palleschi G, Silvestri L, Cavallaro G, Rizzello M, Silecchia G, et al. Pure intracorporeal laparoscopic radical cystectomy with orthotopic "U" shaped ileal neobladder. BMC Urol 2014;14:89.
9. Shao P, Li P, Ju X, Qin C, Li J, Lv Q, et al. Laparoscopic radical cystectomy with intracorporeal orthotopic ileal neobladder: technique and clinical outcomes. Urology 2015;85:368-73.

10. Xu P, Chen B, Xu A, Yuan D, Zhang Y, Liu C. Initial experience with intracorporeal laparoscopic radical cystectomy and detaenial sigmoid neobladder reconstruction. Eur Urol 2021;79:545-51. 